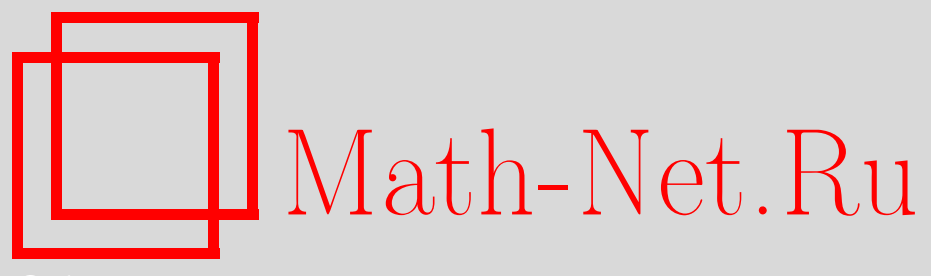

Я. Т. Султанаев, О. В. Мякинова, Об индексах дефекта сингулярного дифференциального оператора четвертого порядка в пространстве вектор-функций, Матем. заметки, 2009, том 86, выпуск 6, 950-953

DOI: https://doi.org/10.4213/mzm8530

Использование Общероссийского математического портала Math-Net.Ru подразумевает, что вы прочитали и согласны с пользовательским соглашением http: //www . mathnet.ru/rus/agreement

Параметры загрузки:

IP: 54.197 .217 .227

26 апреля 2023 г., 10:42:27

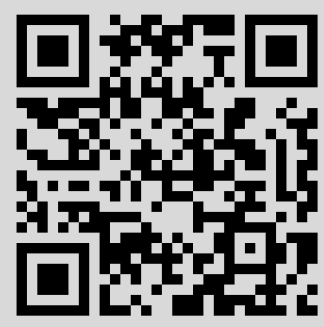




\section{Об индексах дефекта \\ сингулярного дифференциального оператора четвертого порядка в пространстве вектор-функций}

\section{Я. Т. Султанаев, О.В. Мякинова}

Исследованию индексов дефекта обыкновенных дифференциальных операторов посвящено значительное число работ (см. [1], [2] и библиографию к ним). Однако в этих работах, как правило, исследуются скалярные дифференциальные операторы.

В недавних работах Исмагилова и Костюченко [3], [4], посвященных исследованию спектральных свойств неполуограниченных дифференциальных операторов в пространстве вектор-функций, отмечено практическое отсутствие результатов об индексах дефекта таких операторов. В настоящей работе мы исследуем индексы дефекта сингулярного дифференциального оператора четвертого порядка в пространстве вектор-функций.

Рассмотрим в пространстве

$$
H=L^{2}(0,+\infty) \oplus L^{2}(0,+\infty)
$$

минимальный дифференциальный оператор $L_{0}$, порожденный выражением

$$
l(y)=-y^{(4)}+Q(x) y,
$$

$y=\left(y_{1}(x), y_{2}(x)\right)^{T}, 0<x<+\infty$. Здесь $Q(x)=\left\|q_{i, j}(x)\right\|_{i, j=1}^{2}$ - вещественная симметрическая матрица, собственные значения которой

$$
\mu_{1,2}=\frac{q_{11}+q_{22} \pm \sqrt{\left(q_{11}-q_{22}\right)^{2}+4 q_{12}^{2}}}{2}
$$

$\left|\mu_{i}(x)\right| \rightarrow \infty$ при $x \rightarrow+\infty$. Функция $\phi^{\prime}(x)$ - это скорость вращения собственных векторов матрицы $Q(x)$, где $\phi(x)$ определяется по формуле

$$
\phi(x)=\frac{1}{2} \operatorname{arcctg} \frac{q_{22}-q_{11}}{2 q_{12}} .
$$

Следуя работе [3], назовем случай, рассматриваемый в теореме 1 случаем умеренного вращения собственных векторов матрицы $Q(x)$, а случай теоремы 2 - случаем быстрого вращения. Нами доказаны

Теорема 1. Пусть выполнены следующие условия: существует $x_{0}$ такое, что для всех $x>x_{0}$

1) $\left|\phi^{\prime}(x)\right|<$ const;

2) $0<A \leqslant\left|\mu_{i} / \mu_{j}\right| \leqslant B, i, j=1,2$;

3) имеют место оченки

$$
\int_{x_{0}}^{\infty}\left|\mu_{i}\right|^{-1 / 4} d x<\infty, \quad \int_{x_{0}}^{\infty}\left|\frac{\mu_{i}^{\prime 2}}{\mu_{i}^{9 / 4}}+\frac{\mu_{i}^{\prime \prime}}{\mu_{i}^{5 / 4}}\right| d x<\infty, \quad \int_{x_{0}}^{\infty}\left|\frac{\phi^{\prime \prime}}{\mu_{i}^{1 / 4}}\right| d x<\infty, \quad i=1,2 ;
$$

4) $\left|\mu_{i}^{\prime}(x)\right| \leqslant c\left|\mu_{i}(x)\right|^{\alpha}, c=$ const, $i=1,2,0<\alpha<5 / 4$.

Работа выполнена при поддержке Российского фонда фундаментальных исследований (грант № 08-01-97026).

(C) Я. Т. Султанаев, О.В. Мякинова, 2009 
Тогда система $l(y)=\lambda y$ имеет восемь линейно независимых решений $y_{j}(x, \lambda)$ таких, что при $x \rightarrow \infty$

$$
\begin{array}{ll}
y_{1,2}=\psi_{1}(x, \lambda) e^{ \pm \int_{0}^{x}\left(\lambda-\mu_{1}\right)^{1 / 4} d t}(1+o(1)), & y_{3,4}=\psi_{1}(x, \lambda) e^{ \pm i \int_{0}^{x}\left(\lambda-\mu_{1}\right)^{1 / 4} d t}(1+o(1)), \\
y_{5,6}=\psi_{2}(x, \lambda) e^{ \pm \int_{0}^{x}\left(\lambda-\mu_{2}\right)^{1 / 4} d t}(1+o(1)), & y_{7,8}=\psi_{2}(x, \lambda) e^{ \pm i \int_{0}^{x}\left(\lambda-\mu_{2}\right)^{1 / 4} d t}(1+o(1)),
\end{array}
$$

где

$$
\psi_{1}=\frac{1}{\sqrt[8]{\left(\lambda-\mu_{1}\right)^{3}}}\left(\begin{array}{c}
\cos \phi \\
-\sin \phi
\end{array}\right), \quad \psi_{2}=\frac{1}{\sqrt[8]{\left(\lambda-\mu_{2}\right)^{3}}}\left(\begin{array}{c}
\sin \phi \\
\cos \phi
\end{array}\right) .
$$

Теорема 2. Пусть выполнены следующие условия: $\mu_{i}(x) \rightarrow-\infty$ nри $x \rightarrow+\infty$ и существует $x_{0}$ такое, что для всех $x>x_{0}$

1) $\left|\mu_{i}^{\prime}(x)\right| \leqslant C_{1}\left|\mu_{i}(x)\right|^{\alpha}, C_{1}=$ const, $i=1,2,0<\alpha<5 / 4$;

2) $0<A \leqslant\left|\mu_{i} / \mu_{j}\right| \leqslant B, i, j=1,2$;

3) имеют место оценки

$$
\int_{x_{0}}^{\infty}\left|\mu_{i}\right|^{-1 / 2} d x<\infty, \quad \int_{x_{0}}^{\infty}\left|\frac{\mu_{i}^{\prime \prime}}{\mu_{i}^{2}}\right| d x<\infty, \quad \int_{x_{0}}^{\infty}\left|\frac{\phi^{\prime \prime}}{\mu_{i}^{7 / 4}}\right| d x<\infty, \quad i=1,2 ;
$$

4) $\left|\mu_{i} / \phi^{\prime}(x)\right| \leqslant C_{2}, C_{2}=$ const, $i=1,2$.

Тогда система $l(y)=\lambda y$ имеет восемь линейно независимых решений $y_{j}(x, \lambda)$ таких, что при $x \rightarrow \infty$

$$
\begin{array}{ll}
y_{1,2}=\widetilde{\psi}_{1}(x, \lambda) e^{\int_{0}^{x} m^{1 / 4} d t}(1+o(1)), & y_{3,4}=\widetilde{\psi}_{1}(x, \lambda) e^{i \int_{0}^{x} m^{1 / 4} d t}(1+o(1)), \\
y_{5,6}=\widetilde{\psi}_{2}(x, \lambda) e^{-\int_{0}^{x} m^{1 / 4} d t}(1+o(1)), & y_{7,8}=\widetilde{\psi}_{2}(x, \lambda) e^{-i \int_{0}^{x} m^{1 / 4} d t}(1+o(1)),
\end{array}
$$

где

$$
\widetilde{\psi}_{1}=\frac{1}{\sqrt[8]{m^{3}}}\left(\begin{array}{c}
1 \\
\pm i
\end{array}\right), \quad \widetilde{\psi}_{2}=\frac{1}{\sqrt[8]{m^{3}}}\left(\begin{array}{c}
1 \\
\pm i
\end{array}\right), \quad m=\frac{\mu_{1}+\mu_{2}-2 \lambda}{2} .
$$

Наметим схему доказательства. Если ввести обозначение $z=\left(y, y^{\prime}, y^{\prime \prime}, y^{\prime \prime \prime}\right)^{T}$, то перейдем к системе дифференциальных уравнений первого порядка $z^{\prime}=A z$,

$$
A=\left(\begin{array}{cccc}
0 & I & 0 & 0 \\
0 & 0 & I & 0 \\
0 & 0 & 0 & I \\
-Q+\lambda I & 0 & 0 & 0
\end{array}\right)
$$

где $I$ - единичная матрица второго порядка. Далее произведем замену

$$
z=\underbrace{\operatorname{diag}\left\{U_{1}, U_{1}, U_{1}, U_{1}\right\}}_{U} w
$$

где $U_{1}(x)$ - ортогональная матрица

$$
U_{1}=\left(\begin{array}{cc}
\cos \phi & \sin \phi \\
-\sin \phi & \cos \phi
\end{array}\right)
$$

Получим систему уравнений

$$
w^{\prime}=U^{-1} A U w+\underbrace{U^{-1} U^{\prime}}_{P} w .
$$


В первом случае матрицу $U^{-1} A U$ приведем к диагональному виду. Известно, что существует ортогональная матрица $C(x, \lambda)$ такая, что

$$
C^{-1}\left(U^{-1} A U\right) C=M,
$$

где $M$ - диагональная матрица с элементами

$$
\begin{array}{cccc}
\widetilde{\mu}_{1}(x, \lambda)=\sqrt[4]{\lambda-\mu_{1}}, & \widetilde{\mu}_{2}(x, \lambda)=-\sqrt[4]{\lambda-\mu_{1}}, & \widetilde{\mu}_{3}(x, \lambda)=i \sqrt[4]{\lambda-\mu_{1}}, \\
\widetilde{\mu}_{4}(x, \lambda)=-i \sqrt[4]{\lambda-\mu_{1}}, & \widetilde{\mu}_{5}(x, \lambda)=\sqrt[4]{\lambda-\mu_{2}}, & \widetilde{\mu}_{6}(x, \lambda)=-\sqrt[4]{\lambda-\mu_{2}}, \\
\tilde{\mu}_{7}(x, \lambda)=i \sqrt[4]{\lambda-\mu_{2}}, & \widetilde{\mu}_{8}(x, \lambda)=-i \sqrt[4]{\lambda-\mu_{2}} .
\end{array}
$$

Матрицу $C$ можно выбрать так, чтобы выполнялось условие

$$
\left(C^{-1} C^{\prime}\right)_{i i}=0, \quad i=1, \ldots, 8 .
$$

Тогда половина элементов матрицы $C$ равны нулю, а остальные элементы имеют вид

$$
a\left(\lambda-\mu_{k}\right)^{\gamma}, \quad k=1,2, \quad \gamma= \pm \frac{1}{8}, \pm \frac{3}{8}, \quad a= \pm 1, \pm i .
$$

Положим

$$
w=C(I+G) u
$$

где матрица $G$ с элементами $g_{i j}$ удовлетворяет соотношению

$$
G M-M G=C^{-1} C^{\prime}+C^{-1} P C .
$$

После этой замены мы придем к системе уравнений

$$
u^{\prime}=(M+\Theta(x, \lambda)) u
$$

где

$$
\Theta(x, \lambda)=(I+G)^{-1}\left(C^{-1} C^{\prime} G-G^{\prime}+C^{-1} P C G\right) .
$$

Во втором случае в системе (2) приводим к диагональному виду матрицу $P$ заменой $w=T u, T^{-1} P T=\Lambda$,

$$
\Lambda=\operatorname{diag}\left\{\phi^{\prime}(x) i,-\phi^{\prime}(x) i, \phi^{\prime}(x) i,-\phi^{\prime}(x) i, \phi^{\prime}(x) i,-\phi^{\prime}(x) i, \phi^{\prime}(x) i,-\phi^{\prime}(x) i\right\} .
$$

Матрица $T$ постоянная, поэтому $T^{\prime}=0$. Получим систему

$$
u^{\prime}=(\underbrace{T^{-1} U^{-1} A U T}_{C}-\Lambda) u,
$$

где

$$
\begin{gathered}
C=\underbrace{\left(\begin{array}{cccc}
0 & I & 0 & 0 \\
0 & 0 & I & 0 \\
0 & 0 & 0 & I \\
M_{1} & 0 & 0 & 0
\end{array}\right)}_{C_{1}}+\underbrace{\left(\begin{array}{cccc}
0 & 0 & 0 & 0 \\
0 & 0 & 0 & 0 \\
0 & 0 & 0 & 0 \\
M_{2} & 0 & 0 & 0
\end{array}\right)}_{C_{2}}, \\
M_{1}=\left(\begin{array}{cc}
m & 0 \\
0 & m
\end{array}\right), \quad M_{2}=\left(\begin{array}{cc}
0 & \frac{\mu_{1}-\mu_{2}}{2} \\
\frac{\mu_{1}-\mu_{2}}{2} & 0
\end{array}\right) .
\end{gathered}
$$

Приведем матрицу $C_{1}$ к диагональному виду заменой $u=S v, S^{-1} C_{1} S=\Lambda_{1}$,

$$
\Lambda_{1}=\operatorname{diag}\{\sqrt[4]{m}, \sqrt[4]{m}, i \sqrt[4]{m}, i \sqrt[4]{m},-\sqrt[4]{m},-\sqrt[4]{m},-i \sqrt[4]{m},-i \sqrt[4]{m}\}
$$


Как и в первом случае, выберем матрицу $S$ так, чтобы выполнялось условие

$$
\left(S^{-1} S^{\prime}\right)_{i i}=0, \quad i=1, \ldots, 8 .
$$

Тогда половина ее элементов - нули, а остальные элементы имеют вид

$$
m^{k}, \quad k= \pm \frac{3}{8}, \pm \frac{1}{8} .
$$

Обозначим $\Lambda_{1}-\Lambda=\tilde{\Lambda}$. Положим $v=(I+G) t$, где матрица $G$ с элементами $g_{i j}$ удовлетворяет соотношению

$$
G \widetilde{\Lambda}-\widetilde{\Lambda} G=-S^{-1} S^{\prime}+S^{-1} C_{2} S .
$$

После этой замены мы придем к системе уравнений

$$
t^{\prime}=\left(M+\Theta_{1}(x, \lambda)\right) t
$$

где

$$
\Theta_{1}(x, \lambda)=(I+G)^{-1}\left(-S^{-1} S^{\prime} G-G^{\prime}+S^{-1} C_{2} S G\right) .
$$

Далее показывается, что системы (4) и (5) являются $L$-диагональными (см. $[1 ;$ с. 304$])$. Асимптотика решений этих систем известна (см. [5; с. 224]). Возвращаясь с помощью обратных замен к вектору $y$, получим требуемые асимптотические формулы.

Из асимптотических формул для решений системы $l y=\lambda y$ можно сделать вывод об индексах дефекта оператора $L_{0}$.

Теорема 3. Пусть выполнены все условия теоремы 1. Тогда

1) если $\mu_{i} \rightarrow+\infty, i=1,2$, то индексы дефекта оператора $L_{0}$ равны $(4,4)$;

2) если $\mu_{i} \rightarrow-\infty, i=1,2$, то индексы дефекта оператора $L_{0}$ равны $(6,6)$;

3) если $\mu_{i} \rightarrow+\infty, \mu_{j} \rightarrow-\infty, i, j=1,2, i \neq j$, то индексы дефекта оператора $L_{0}$ равны $(5,5)$.

Утверждения теоремы следуют из того, что в первом случае половина показателей экспоненты имеет положительную вещественную часть, а другая половина - отрицательную вещественную часть. Во втором случае при больших $x$ два показателя экспоненты будут чисто мнимыми.

Теорема 4. Пусть выполнены все условия теоремы 2. Тогда индексы дефекта оператора $L_{0}$ равны $(4,4)$.

\section{СПИСОК ЦИТИРОВАННОЙ ЛИТЕРАТУРЫ}

[1] А.Г. Костюченко, И.С. Саргсян, Распределение собственных значений. Самосопряженные обыкновенные дифференииальные операторы, Наука, М., 1979. [2] M.S.P. Eastham, The Asymptotic Solution of Linear Differential Systems. Applications of the Levinson Theorem, London Math. Soc. Monogr. (N.S.), 4, Clarendon Press, Oxford, 1989. [3] Р. С. Исмагилов, А.Г. Костюченко, Функи. анализ и его прил., 42:2 (2008), 11-22. [4] Р. С. Исмагилов, А. Г. Костюченко, Функи. анализ и его прил., 41:1 (2007), 39-51. [5] М. А. Наймарк, Линейнъе дифференииалъные операторы, Наука, М., 1969.

\section{Я. Т. Султанаев}

E-mail: SultanaevYT@bsu.bashedu.ru

\section{О. В. Мякинова}

Башкирский государственный университет, г. Уфа

E-mail: llmmst@rambler.ru 